\title{
Development and evaluation of VIE-PNN, a knowledge-based system for calculating the parenteral nutrition of newborn infants
}

\author{
Werner Horn $^{\text {a,b }}$, Christian Popow $^{c}$, Silvia Miksch ${ }^{\text {d,b }}$, \\ Lieselotte Kirchner ${ }^{\mathrm{c}}$, Andreas Seyfang ${ }^{\mathrm{d}, \mathrm{a}}$ \\ ${ }^{\mathrm{a}}$ Department of Medical Cybernetics and Artificial Intelligence, University of Vienna \\ Freyung 6, A-1010 Vienna, Austria \\ E-mail:werner@ai.univie.ac.at \\ ${ }^{\mathrm{b}}$ Austrian Research Institute for Artificial Intelligence \\ ${ }^{\mathrm{c}}$ NICU, Division of Neonatology, Department of Pediatrics, University of Vienna \\ ${ }^{\mathrm{d}}$ Institute of Software Technology, Vienna University of Technology
}

\begin{abstract}
Calculating the daily changing composition of parenteral nutrition for small newborn infants is troublesome and time consuming routine work in neonatal intensive care. The task needs expertise and experience and is prone to inherent calculation errors. We designed VIE-PNN, a knowledge-based system in order to reduce daily routine work and calculation errors. VIE-PNN was redesigned several times because the clinicians accepted the system only when it saved time. The most recent version of VIE-PNN uses a HTML-based clientserver architecture and is integrated into the intranet of the local patient data management system. Since more than three years all parenteral nutrition plans are calculated using VIEPNN. Evaluating the system's performance and the users contentedness, we compared 50 nutrition plans calculated in parallel using VIE-PNN or a hand-held calculator, retrospectively analyzed more than 5000 nutrition plans stored in VIE-PNNs database and evaluated a user questionnaire. Nutrition plans were calculated in a mean time of 2.4 vs. 7.1 minutes using VIE-PNN or the hand-held calculator. Errors and omissions in the nutrition plans were detected in 22 vs. $56 \%$ and errors in the VIE-PNN's plans occurring only with interactively changed values. Reviews of stored plans show that a mean of 4 out of 16 parameters were interactively changed. VIE-PNN was well accepted. Most important reasons for the successful operation of VIE-PNN in the daily routine work were time savings and robustness of the system.
\end{abstract}

Key words: Knowledge-based system, intensive care unit, parenteral nutrition, neonates, intranet application, integration with patient data management system 


\section{Introduction}

The planning and calculation of an adequate nutritional support for meeting the metabolic requirements of sick or premature neonates is an important daily routine task. It is time consuming, needs practical expert knowledge and experience, and involves the risk of introducing possibly fatal errors $[5,7,15,16]$. The daily time required per patient is approximately 10 minutes summing up to more than one hour for a eight beds neonatal intensive care unit (NICU). Approximately 5-50\% of all parenteral nutrition solution (PNS) sheets contain calculation or omission errors requiring recalculation or even remixture of the nutrition solution. This is costly and requires additional time of physicians and nurses.

Computer programs supporting the calculation of parenteral nutrition plans are available at a few hospitals. Most of them are designed for adult or pediatric use (e.g., $[10,14]$ ) without giving specific consideration to the specific needs of newborn infants. There are a few pediatric programs for parenteral nutrition planning covering also the group of newborn and premature infants $[6,11,12]$.

Such programs, however, do not cover the specific metabolic and nutritional needs of extreme low birth-weight newborn infants (birth-weight 500-1000 grams). Only a few programs were specifically tailored to the needs of small newborn infants: there are simple calculation programs $[2,3,8,11]$ and more complex rule-based and knowledge-based systems $[4,6]$. Both types of programs reduce the routine work load, and lead to a more optimized nutrition supply. Most systems are tailored to the needs of a specific neonatal intensive care unit, and their portability to other units is very limited because of differences in the clinical approach (policy of fluid supply, speed of increments, inclusion or exclusion of various minor nutrients, exactness of calculations, availability of various solutions and brands etc.)

We designed VIE-PNN (Vienna Expert System for Parenteral Nutrition of Neonates) aiming to create a program for the routine support of our NICU combining standard textbook knowledge about neonatal nutrition with the practical knowledge of expert neonatologists.

In the year 1991 we started the implementation of VIE-PNN with the following main goals:

- creating an interactive support system for calculating the daily nutritional plans (PNS, parenteral nutrition solution),

- reducing the time needed for the calculation,

- creating a system able to combine parenteral and enteral feedings,

- providing a continuous increase of the daily nutrition supply and a forced change from parenteral to oral nutrition. This requires reasoning methods taking into account the data of the previous days,

- output of a printed PNS schedule to become part of the case history. The PNS 
Table 1

Input and output parameters of VIE-PNN.

\begin{tabular}{lll}
\hline Input parameters & Output parameters & Statistical measurements \\
\hline Body weight & Parenteral supply & Energy rates \\
Age & Enteral supply & Parenteral infusion rate \\
Total fluid allowance & Glucose & Fat infusion rate \\
Enteral products & Proteins / amino acids & \\
Drugs & Electrolytes & \\
Serum values & Vitamins & \\
Clinical problems & Trace elements \\
& Fat \\
& Bypass medication & \\
\hline
\end{tabular}

schedule is further used by the nurses to compose the daily mixture of the nutrition solution,

- maintaining the hospital's standard for neonatal nutrition management and providing adequate control of fluid, electrolyte, fat, nitrogen, and caloric needs. The standard is defined in terms of rules of expert neonatologists, and

- enabling the experts to keep the rules up-to-date.

\section{The VIE-PNN system}

VIE-PNN consists of three main modules: patient administration, computation of the daily PNS schedule, and maintenance of the knowledge base. Patient administration is needed for adding new patients (name, sex, date of birth, venous access) and for archiving the data of discharged infants. The maintenance module supports editing of nutrition rules, parameter limits, rounding factors, and updating of oral nutrition preparations.

The main module of VIE-PNN interactively calculates the daily PNS schedule. It starts with yesterday's PNS values of the infant (or plausible default values at the day of hospital admission). These values determine the input parameters of the system (see table 1).

VIE-PNN determines the PNS schedule by

- applying rules for the daily increase of nutrition components,

- applying rules for nutrition needs based on today's serum values,

- applying rules for adapting the nutritional requirements to the clinical problems of the infant, 
- decreasing parenteral nutrition components by the ratio of enteral to total supply (for components which are contained in oral products),

- checking for range limits of the components,

- applying rounding methods based on knowledge about the precision needed for each component,

- combining drugs for bypass medication,

- solving the problem if the total fluid allowance is too small to cover the nutritional requirements.

A sample rule is given in Figure 1. It determines the daily prescription of glucose based on age, actual serum glucose concentration and the former glucose supply. VIE-PNN's rules are applied in a forward chaining manner to determine today's values of all output parameters.

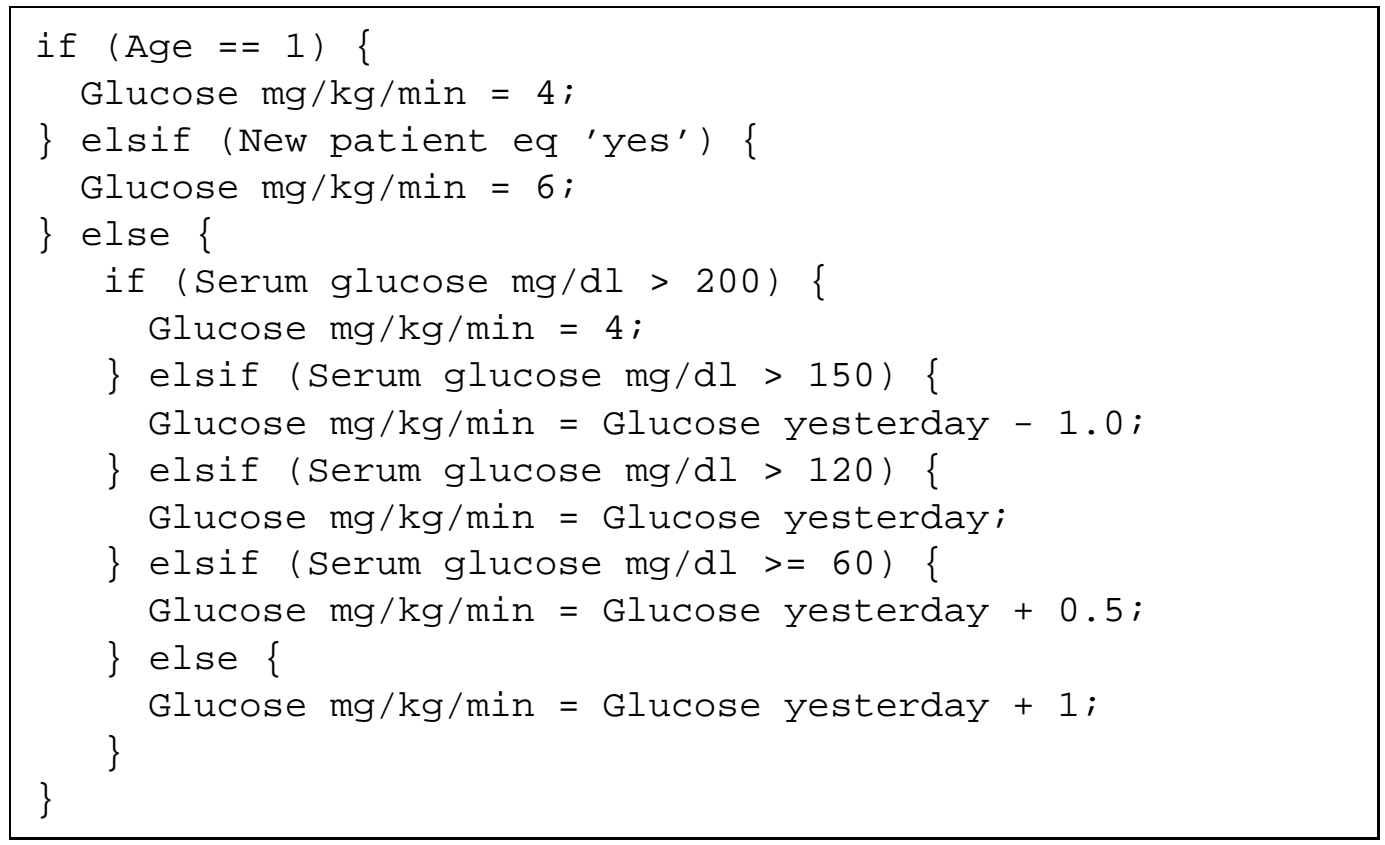

Fig. 1. Sample VIE-PNN rule: daily glucose prescription.

The final PNS schedule consists of the output parameters shown in table 1. VIEPNN is interactive allowing the physician to modify each of the input and output parameters.

The first four versions of VIE-PNN [9] were stand-alone PC versions. The initial three versions were implemented using an expert system shell ("Intelligence/Compiler $(\mathrm{I} / \mathrm{C})$ "). These versions were too slow to be useful in daily routine. The fourth version was a redesign in $\mathrm{C}++$ with a user interface written in TurboVision. It was fast, but inflexible. It was not accepted by most of the clinicians due to the rigid way the user interface behaved. Furthermore, it was impossible for the experts to adapt VIE-PNN's decision rules to changing nutritional standards. Such changes in the knowledge base required source code changes by the knowledge engineers and recompilation of the system. The system was introduced at the NICU including 
some initial training. It was evaluated successfully for its correctness [1] but was used by only a few physicians and then not on a regular basis. The main reasons were its inflexible user interface and its installation on a stand-alone PC: The PC was located in a room off-site and it was often used for other urgent tasks.

\section{Integrating VIE-PNN into the clinical intranet}

The negative experiences with the stand-alone version of VIE-PNN motivated us to try a completely different approach. The NICU is equipped with an intranet of workstations. The workstations (two mirrored servers, a bedside workstation for each bed, and several workstations in the nurses' and the physicians' rooms) run the patient data management system (PDMS) Hewlett-Packard CareVue 9000. All patient records are handled electronically by this PDMS. Our goal was to integrate VIE-PNN into this system. Unfortunately, HP CareVue is a completely closed system. There was no possibility to integrate knowledge-based modules which would be the ideal solution for a parenteral nutrition support system. We were therefore forced to choose the second best solution: using the workstations as a display for our VIE-PNN system. We added an additional server to the intranet which runs VIE-PNN on request of any client workstation.

The redesign of VIE-PNN (version 5) for a client-server architecture is based on the Hypertext Markup Language (HTML). A button in the HP CareVue system allows to invoke an HTML browser on any of the client workstations. This browser sends requests to the HTTP server of the VIE-PNN server workstation. All the reasoning and calculations are done by cgi-programs written in the programming language PERL. Requests for data input and results of VIE-PNN are sent to the client workstation in the form of HTML pages. The final PNS schedule is printed on the NICU's laser printer. The system's knowledge is coded in PERL-like IFTHEN rules. It is revisable by the experts using the HTML browser.

Using eight HTML pages VIE-PNN asks for input and verification of the nutritional parameters. Minimal input is required from the physician. Manual input is mandatory for serum values from the laboratory (if new values are available), for changes in the amount or kind of oral feeding and for changes in drug administration (bypass medication). If there are no changes the physician may accept all pages by clicking the OK button. The system recommends values for nutritional components based on its knowledge rules. Figure 2 shows such a page for electrolyte values. The physician may change a value in case the nutrition requirements differ from standard rules due to a problem of the neonate which is not included in the knowledge base of VIE-PNN. Pressing the I(nformation) button gives an in-depth explanation about the computation of the recommended value (including basic values, rounding factors, and rules used). The final page shows the complete PNS schedule (see Figure 3). The user may accept it and print the PNS sheet or 


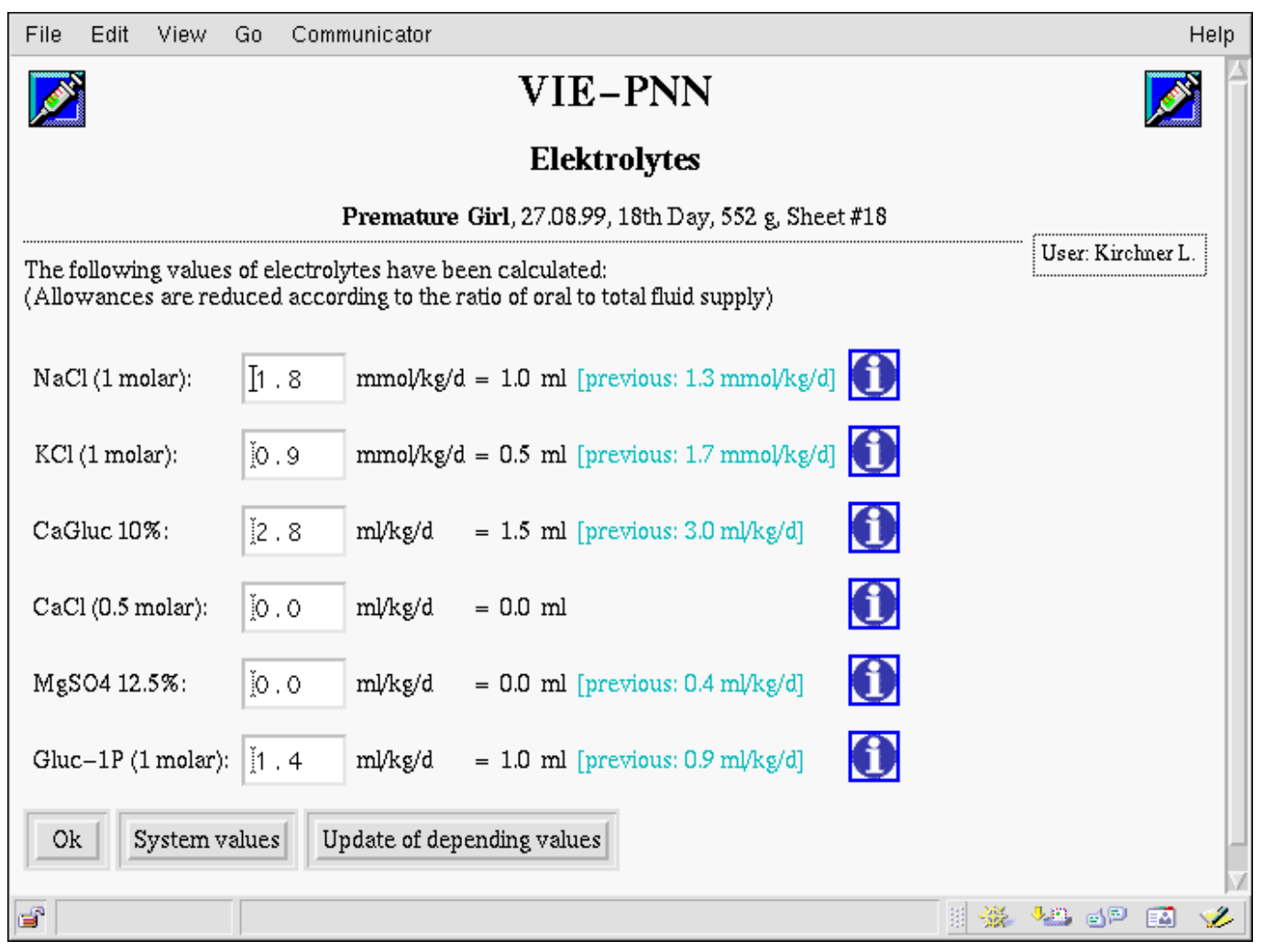

Fig. 2. Sample page showing recommendations of VIE-PNN for supplying electrolytes. The physician may change the values. The I(nformation) button activates an explanation page with details about the computation of electrolyte supply.

again modify single parameters.

One of the most important features of knowledge-based systems is the ease of maintenance. Expert neonatologists must have good support to keep the knowledge about oral products, drugs, plausible limits of parameters and prescription rules up-to-date. VIE-PNN has a HTML-based maintenance module which allows the supervising neonatologist to edit the knowledge. Figure 4 shows the menu of possible maintenance actions available to the physician (left). Using a rule editor he/she may alter prescription rules (right). Such changed rules are checked for correct syntax and consistency. If the validation succeeds they are converted to PERL code and put in use immediately.

\section{Routine use and evaluation of VIE-PNN}

We installed the client-server intranet system of VIE-PNN at the NICU by end of June 1996. It was immediately accepted by the physicians of the NICU. Only a few minutes of initial training were required. After two years of successful operation VIE-PNN was installed at a second NICU of the University of Vienna Medical 


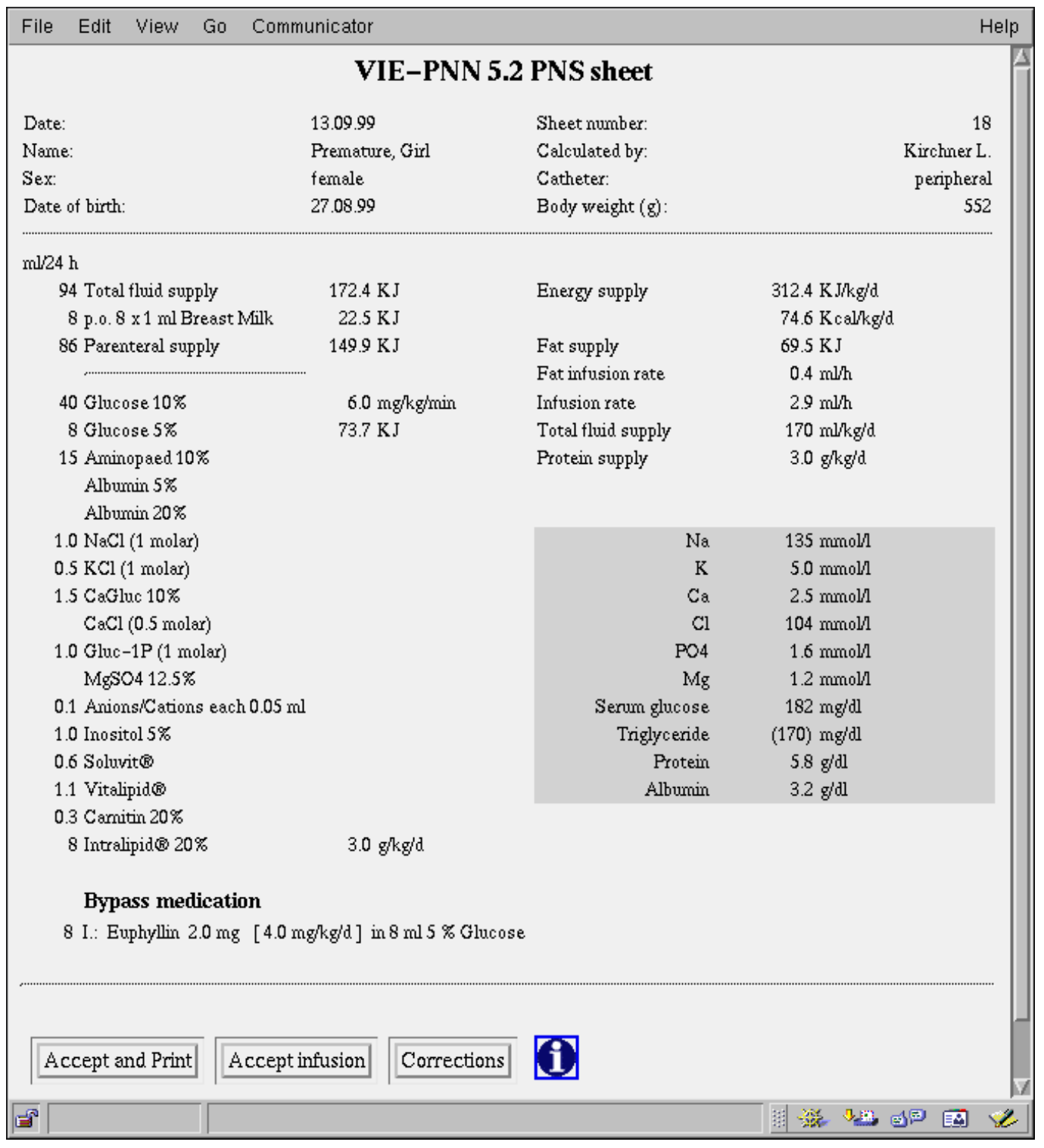

Fig. 3. VIE-PNN's HTML page showing the final composition of the parenteral nutrition solution. The top part gives administrative data. The left column shows the components of the PNS. The right column gives statistical measurements and today's serum values.

School. VIE-PNN is now in continuous operation at these two NICUs. It calculates approximately 5000 nutrition sheets per year. There are no more PNS sheets calculated by hand. We have not received any complaints about errors in the PNS sheets. One may suspect that this is due to the interactiveness of the system: the physician eliminates all erroneous computations of the system. However, we have no reports about changes required on standard cases. We are convinced that knowledge-based systems like VIE-PNN have to be interactive. There is no possibility to cover all possible extreme situations which require a modification of the common nutrition schedule.

The continuous operation at two NICUs over years underwrites that VIE-PNN is 

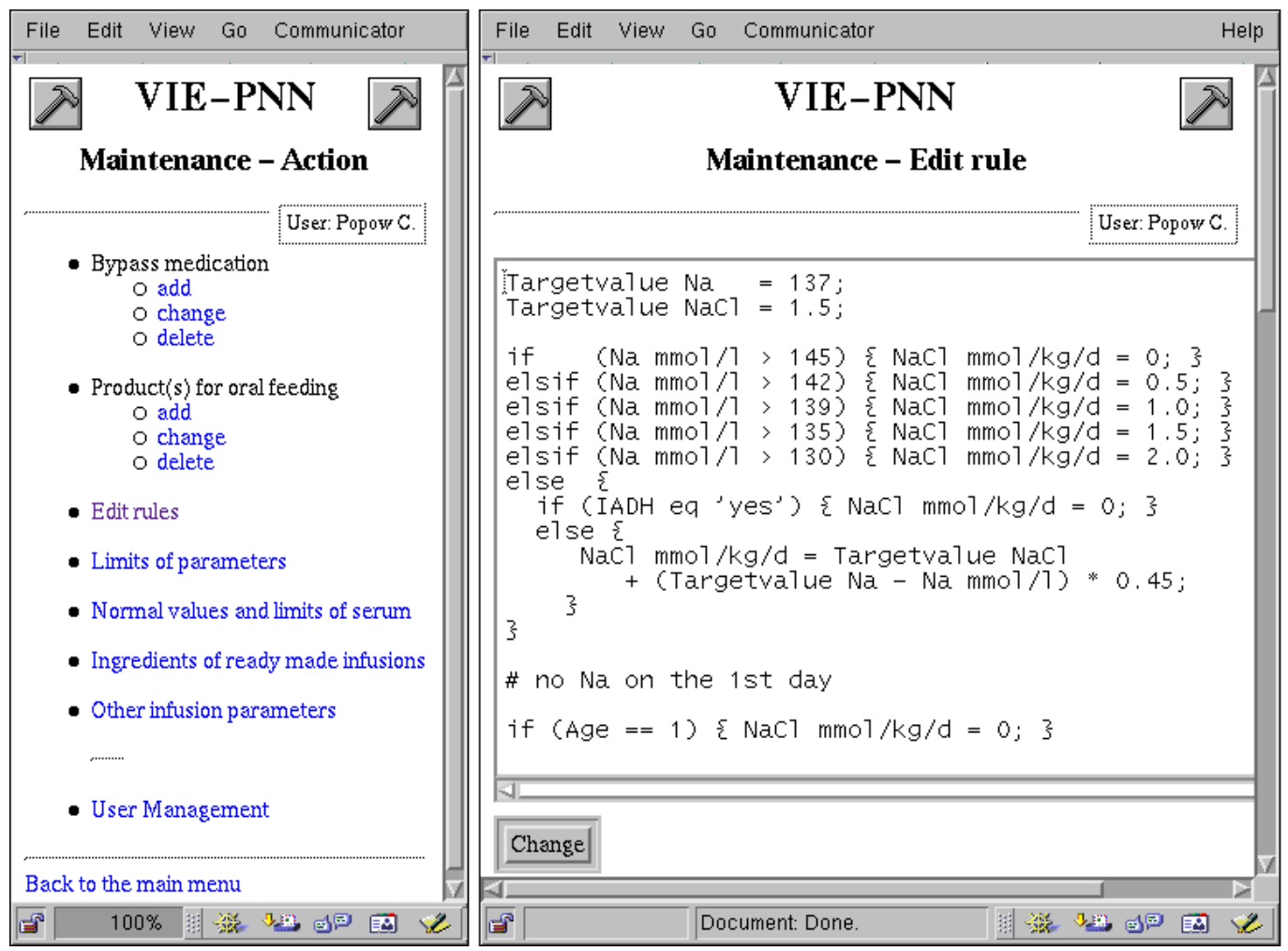

Fig. 4. VIE-PNN maintenance: (left) the menu of possible maintenance actions, (right) the rule editor with the rule for sodium prescription.

trusted and used in the daily routine. Failure of the computer system disabling VIEPNN is hardly tolerated by the clinicians. We nevertheless wanted to examine the performance of VIE-PNN more closely. After two years of operation we performed a clinical study to evaluate the system and its performance:

- we prospectively evaluated 50 routine PNS calculated in parallel by using VIEPNN or by the traditional way using a hand-held calculator. Both calculations were performed by various physicians in charge,

- we retrospectively reviewed the PNS stored in the systems database for two years (July 1996 to 1998) for the number of interactively changed parameters and necessary program updates, and

- we evaluated a questionnaire distributed to all neonatologists working with the system.

For the 50 routine PNS we compared the time needed for calculating a PNS prescription and the differences in the amount of PNS components exceeding more than one unit (e.g., $1 \mathrm{mmol} / \mathrm{kg} \mathrm{Na}, 0.5 \mathrm{~g} / \mathrm{kg}$ amino acids). Both PNS sheets were later reviewed by a senior neonatologist who rated errors and omissions as potentially life threatening (like prescribing dangerous concentrations of potassium or bypass medication), major (e.g., prescribing too little amounts of calcium which is needed for bone mineralization) or minor or clinically not relevant (like forgetting to calculate the total energy supply). 
The users questionnaire asked for the frequency of system use, the degree of contentedness with the system (on a 5 grade scale), various reasons for using the system, and for desired modifications. The questionnaire was made available to all attending physicians. It was answered by all 11 physicians of the NICU.

We found significant ( $p<0.001$, paired t-test) time savings of a mean of 4.7 minutes per PNS prescription (see Figure 5). The mean time needed to calculate a PNS was 2.4 minutes (VIE-PNN) vs. 7.1 minutes (hand-held calculator). This sums up to about $\frac{1}{2}$ to 1 hour daily time savings for 8-12 PNS prescriptions.

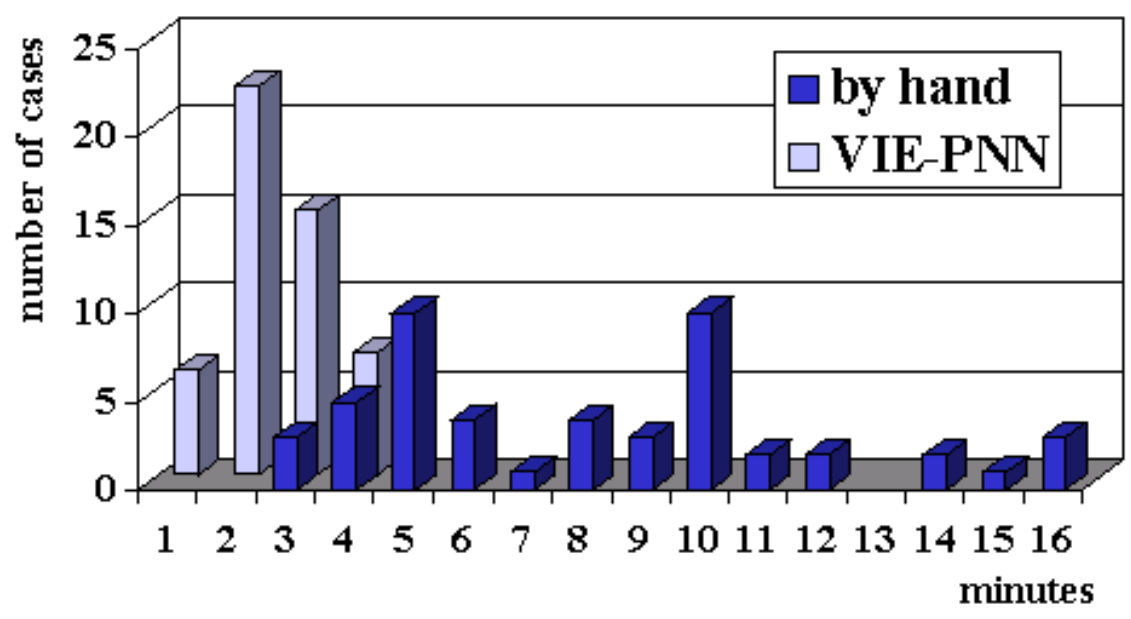

Fig. 5. Comparison of time physicians needed to calculate a parenteral nutrition solution using a hand-held calculator versus using VIE-PNN.

There were only little and no systematic differences in the composition of the PNS and no life threatening errors. There were less major (10\% vs. 18\%) and minor (12\% vs. 66\%) errors and omissions in the VIE-PNN calculated prescriptions. Major errors were related to prescribing glucose (0\% vs.10\%), electrolytes (10\% vs. $6 \%$ ) or bypass medication ( $0 \%$ vs. $2 \%)$, minor errors to prescribing glucose $(0 \%$ vs. $14 \%)$, fat (6\% vs. $2 \%)$, electrolytes (0\% vs. $16 \%)$, vitamins $(6 \%$ vs. $6 \%$ ) and to forgetting to calculate the energy content of the PNS (0\% vs. 28\%). All errors detected in the VIE-PNN calculated prescriptions were related to values that were interactively changed by the prescribing physician.

Retrospective evaluation of the 5593 PNS of 643 patients stored in the systems database showed that in the mean four out of 16 parameters were interactively modified. Throughout the study period the rules for calculating a PNS were revised or changed for clinical reasons including the rules for prescribing macronutrients (glucose, fat, amino acids), bypass medication and oral nutrition (e.g. adding new brands), and the modification of rounding factors.

The overall rating of the systems performance and usability was good (mean 1.9 on a 5 grade scale, 1 is best). VIE-PNN was mainly used for its time saving (73\%) and accuracy improving $(73 \%)$ features. The attending physicians requested several 
amendments: the implementation of new rules for vitamins and calcium prescription in extreme low birth-weight infants, addition of new bypass medications, and the adaptation of value ranges tolerated and rounding rules. These requests have been easily fulfilled by use of the maintenance module (after discussion with the expert neonatologists.)

\section{Discussion}

The success of the HTML-based system has to be explained mainly by the ease of use and the benefits in terms of time savings and accuracy. Similar benefits were reported by Puangco et al.[13].

Comparing the stand-alone PC version and the client-server version there are no essential changes of the program flow and the knowledge used. Even the final printout of the PNS schedule is the same. Identifiable factors adding to the success of the HTML-based version are:

- VIE-PNN is run in the routine computer environment of the local PDMS;

- the HTML browser is classified as an intuitive user interface. Without any specific training it is immediately clear to the user which box accepts an input, which buttons to press and which links to follow;

- the HTML browser provides the required flexibility for the user. Especially, the BACK and FORWARD buttons allow to review and revise pages;

- the explanation facility (using the I(nformation) button) adds much to the confidence in the conclusions of VIE-PNN. Often a parameter looking unplausible appears correct after looking at the explanation page. An example of such a complicated calculation is the proportional subtraction of components from the parenteral nutrition which are contained in the enteral product. Such a calculation is usually not done by hand;

- most important, physicians benefit from the time savings. This has been verified by the evaluation study. From the subjective physicians' view the savings are dramatical: no one would calculate any more PNS sheets by hand because of the time required.

The integration of VIE-PNN into the intranet of workstations running the patient data management system (PDMS) was an important step in bringing a knowledgebased system into daily routine use. However, it is far from perfect. We would like to integrate the PNS schedule composition as a module into the HP CareVue PDMS. At the moment this is impossible due to the operation of the PDMS as a closed system. More seriously, it is even not possible to get the laboratory values required by VIE-PNN from the PDMS at the required time. There is an export facility in the PDMS which we try to use, but it is too rigid to be useful for data exchange. The automatic inclusion of the ten serum values into VIE-PNN would 
be a great step forward in minimizing manual input. We tried to find solutions for this task by interfacing laboratory equipment.

The current design of VIE-PNN supports portability to other hospitals. It is an encapsulated HTTP-application currently running at two NICUs and at a demo site outside the hospital. It can be installed at every hospital running an HTTP-server (with PERL support for CGIs) and clinical workstations with access to this server. Use of the maintenance module simplifies the customization of prescription rules, enteral products and drugs to local requirements.

A further step has been a reimplementation of VIE-PNN in Java (VIE-PNN 6.0). The whole PNS schedule is computed at the client side using a Java applet. This code includes the current nutrition rules and the PNS data of the previous days. The client applet communicates with a dedicated VIE-PNN server delivering and taking over the PNS data. This version looks promising and saves even more time because data are entered only on three compared to formerly eight screens. Because the stability of the Java solution is still questionable, this new version has, however, not been introduced into the daily routine. In addition, the Java implementation once again will make maintenance a tricky issue.

\section{Lessons learned}

VIE-PNN is a knowledge-based system (KBS) which solves a time-consuming problem in daily routine. It is less the amount and complexity of the knowledge covered which makes it a success, but its ability to implement practical clinical knowledge in the daily routine.

From this success story we have learned the following:

- A system containing textbook and clinical knowledge may be a success even if physicians do have that knowledge at hand. Our experience shows that successful KBS are not restricted to complex domains of application with very specialized knowledge. A KBS is valuable in the hecticness of the clinical routine because it improves the accuracy and the quality of care.

- It is essential to implement practical knowledge. Two examples are the knowledge about the precision needed for each component (expressed in rounding rules), and the coding of support methods for patients where the total fluid allowance it too small to incorporate all the necessary nutritional components. This problem, normally solved by weak estimations, has been very elegantly solved by a manageable stepwise approach. Such practical solutions account for the acceptance and use of a system.

- Explanation facilities are essential for establishing confidence in the system, especially in the first trials. VIE-PNN creates explanations for all its calculations. 
The explanatory pages were particularly often inspected by new users who also wanted to verify their knowledge.

- It is necessary to produce printed output sheets usable as part of the medical record. The electronic storage of all data does not release from the need for a printed output used by physicians and nurses for manual remarks;

- The continuous change of knowledge about nutritional requirements requires a system maintained by expert physicians. A KBS will not be used in the long run if maintenance actions are restricted to knowledge engineers. In addition, physicians will not trust the system if the knowledge is hidden by some (complex) formalism;

- Integration of KBS into clinical software systems (e.g., PDMS) is a necessity for wide-spread use. Even loose integration like using the same hardware as the PDMS is sufficient in the first run. However, a strong integration of all systems including data exchange is the desire for the future;

- Finally, never try to install a system which requires additional time from physicians or nurses. Time savings are the key issue for the success of systems in daily routine use.

VIE-PNN is an example of a successful KBS in daily routine use. First of all, it considerably saves physicians' time. This is achieved by replacing a complex manual computation by a support system which knows about clinical prescription rules. The knowledge contained in VIE-PNN helps the physician to avoid errors and to maintain the high quality standards of neonatal intensive care.

\section{Acknowledgements}

We greatly appreciate the support given to the Austrian Research Institute of Artificial Intelligence (ÖFAI) by the Austrian Federal Ministry of Education, Science and Culture.

\section{References}

[1] M.Dobner, S.Miksch, W.Horn, C.Popow, VIE-PNN: Ein Expertensystem für die Berechnung der parenteralen Ernährung von intensiv behandelten Früh- und Neugeborenen, Wien Klin Wochenschr, 107 (1995) 128-132.

[2] R.Duncan, J.J.Pomerance, Computer Assistance in Delivery of Patient Care in a Neonatal Intensive Care Unit, in: Harris and Bahr, eds., The Use of Computers in Perinatal Medicine (Praeger, New York, 1982) 337.

[3] R.J.Geller, S.A.Blackburn, D.H.Glendon, W.H.Henneman, W.P.Steffee, Computer Optimization of Parenteral Nutrition, J Parenter Ent Nutr, 3 (1979) 79-83. 
[4] R.G.Harper, E.Carrera, S.Weiss, M.Luongo, A Complete Computerized Program for Nutritional Management in the Neonatal Intensive Care Nursery, Am J Perinatol, 2 (1985) 161-162.

[5] M.C.Hermansen. R.Kahler, B.Kahler, Data Entry Errors in Computerized Nutritional Calculations, J Pediatr, 109 (1986) 91-93.

[6] J.Kuchenbecker, L.Urbina, M.Müller, Das weiterentwickelte Computerprogramm PEDINFUS für die totale und additive parenterale Ernährung von Kindern, Infusionsther Transfusionsmed, 23 (1996) 35-40.

[7] J.D.Legler, Physician's Accuracy in Manual Computation, MD Comput, 7 (1990) 155159.

[8] C.Migliori, C.Verdura, C.Belloni, Experience with the Use of a Computerized Data Processing Program on Solutions for Parenteral Nutrition of Neonates, Minerva Pediatr, 49 (1997) 229-234.

[9] S.Miksch, M.Dobner, W.Horn, C.Popow, VIE-PNN: An Expert System for Parenteral Nutrition of Neonates," in: Proceedings of the Ninth Conference on Artificial Intelligence for Applications (CAIA-93), (IEEE Press, New York, 1993) 285-291.

[10] C.Ochoa-Sangrador, M.F.Brezmes-Valdivieso, C.Gil-Valino, Pediatric Parenteral Nutrition Mixtures Design Program: Validity and Stability Study, Comput Biomed Res, 28 (1995) 271-281.

[11] D.Picart, B.Guillois, L.Nevo, D.Alix, A Program for Parenteral and Combined Parenteral and Enteral Nutrition of Neonates and Children in an Intensive Care Unit, Intensive Care Med, 15 (1989) 179-182.

[12] M.Piert, D.Kistler, R.Hettich, Computer-assisted Infusion and Nutrition Planning in an Intensive Care Burn Unit, Intensive Care Med, 15 (1989) 121-125.

[13] M.A.Puangco, H.L.Nguyen, M.J.Sheridan, Computerized PN Ordering Optimizes Timely Nutrition Therapy in a Neonatal Intensive Care Unit, J Am Diet Assoc, 97 (1997) 258-261.

[14] P.R.Schloerb, Electronic Parenteral and Enteral Nutrition, J Parenter Enteral Nutr, 24 (2000) 23-29.

[15] J.J.Tepas, D.L.Mollitt, D.L.String, P.Pieper, Error in Fluid and Calorie Calculation in the Surgical Neonate, J Pediatr Surg, 26 (1991) 132-134.

[16] M.J.Vincer, J.M.Murray, A.Yuill, A.C.Allen, J.R.Evans, D.A.Stinson, Drug Errors and Incidents in a Neonatal Intensive Care Unit, Am J Dis Child, 143 (1989) 737-740. 